\title{
Solving the NLO BK equation in coordinate space
}

\section{T. Lappi*}

Department of Physics, P.O. Box 35, 40014 University of Jyväskylä, Finland

Helsinki Institute of Physics, P.O. Box 64, 00014 University of Helsinki, Finland

E-mail: tuomas.v.v.lappi@jyu.fi

\section{H. Mäntysaari}

Department of Physics, P.O. Box 35, 40014 University of Jyväskylä, Finland

E-mail: heikki.mantysaari@jyu.fi

We present results from a numerical solution of the next-to-leading order (NLO) BalitskyKovchegov (BK) equation in coordinate space in the large $N_{\mathrm{c}}$ limit. We show that the solution is not stable for initial conditions that are close to those used in phenomenological applications of the leading order equation. We identify the problematic terms in the NLO kernel as being related to large logarithms of a small parent dipole size, and also show that rewriting the equation in terms of the "conformal dipole" does not remove the problem. Our results qualitatively agree with expectations based on the behavior of the linear NLO BFKL equation.

XXIII International Workshop on Deep-Inelastic Scattering 27 April - May 12015

Dallas, Texas

\footnotetext{
* Speaker.
} 


\section{Introduction}

The CGC picture [1] of high energy QCD has been succesfully applied to describe deep inelastic scattering, single particle production and particle correlations in forward rapidity protonproton and proton nucleus collisions. It also forms a basis for understanding the initial stage of deconfined QCD matter created in ultrarelativistic nucleus-nucleus collisions. An essential ingredient in these calculations are the JIMWLK equation and its mean field limit - the Balitsky-Kovchegov (BK) equation [2, 3]. They describe the energy, or equivalently Bjorken- $x$, dependence of correlators of Wilson lines in the target color field. Cross sections for different processes are then expressed in terms of these Wilson line correlators.

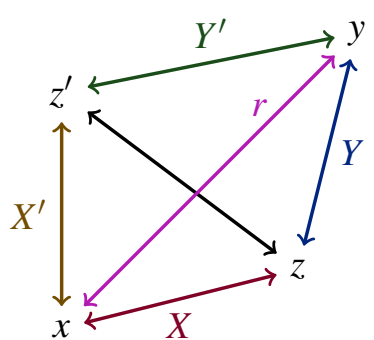

Figure 1: Coordinates for the eikonal Wilson lines and their separations

Recently first steps have been taken to develop this phenomenological picture to next-toleading order (NLO) accuracy, both for the evolution equations themselves $[4,5,6]$ and for specific scattering processes $[7,8,9,10]$. There remain both conceptual and numerical challenges in carrying out the full CGC phenomenology program at NLO accuracy. This talk discusses in more detail one of these aspects, by presenting the result [11] from a direct "brute force" numerical solution of the NLO BK equation as it is written down in Ref. [4].

\section{The equation}

In the large- $N_{\mathrm{c}}$ limit (but assuming $N_{\mathrm{f}} \sim N_{\mathrm{c}}$ ) we can write the evolution equation derived in Ref. [4] for the dipole as:

$$
\begin{aligned}
\partial_{y} S(r) & =\frac{\alpha_{\mathrm{s}} N_{\mathrm{c}}}{2 \pi^{2}} K_{1} \otimes[S(X) S(Y)-S(r)]+\frac{\alpha_{\mathrm{s}}^{2} N_{\mathrm{f}} N_{\mathrm{c}}}{8 \pi^{4}} K_{f} \otimes S(Y)\left[S\left(X^{\prime}\right)-S(X)\right] \\
+ & \frac{\alpha_{\mathrm{s}}^{2} N_{\mathrm{c}}^{2}}{8 \pi^{4}} K_{2} \otimes\left[S(X) S\left(z-z^{\prime}\right) S\left(Y^{\prime}\right)-S(X) S(Y)\right], \quad \text { with } S(r)=\frac{1}{N_{\mathrm{c}}}\left\langle\operatorname{Tr} U^{\dagger}(x) U(y)\right\rangle,
\end{aligned}
$$

Here $U(x)$ is a fundamental representation Wilson line describing the propagation of a high energy probe through the dense color field of the target. The Wilson lines are needed at coordinates $x, y, z, z^{\prime}$ in the two-dimensional transverse plane, with the six distances between them denoted as $r, X, X^{\prime}, Y, Y^{\prime}$ and $z-z^{\prime}$ as shown in Fig. 1 . The convolutions $\otimes$ denote integrations over $z$ (in $K_{1}$ ) or $z$ and $z^{\prime}$ (the other terms). We replace the terms explicitly proportional to the $\beta$-function coefficient in the first kernel $K_{1}$ by the "Balitsky" running coupling prescription [12]. With this replacement the explicit expression for the first kernel is

$$
\begin{aligned}
\frac{\alpha_{\mathrm{s}} N_{\mathrm{c}}}{2 \pi^{2}} K_{1}=\frac{\alpha_{\mathrm{s}}(r) N_{\mathrm{c}}}{2 \pi^{2}}\left[\frac{r^{2}}{X^{2} Y^{2}}+\frac{1}{X^{2}}\right. & \left.\left(\frac{\alpha_{\mathrm{s}}(X)}{\alpha_{\mathrm{s}}(Y)}-1\right)+\frac{1}{Y^{2}}\left(\frac{\alpha_{\mathrm{s}}(Y)}{\alpha_{\mathrm{s}}(X)}-1\right)\right] \\
+ & \frac{\alpha_{\mathrm{s}}(r)^{2} N_{\mathrm{c}}^{2}}{8 \pi^{3}} \frac{r^{2}}{X^{2} Y^{2}}\left[\frac{67}{9}-\frac{\pi^{2}}{3}-\frac{10}{9} \frac{N_{\mathrm{f}}}{N_{\mathrm{c}}}-2 \ln \frac{X^{2}}{r^{2}} \ln \frac{Y^{2}}{r^{2}}\right] .
\end{aligned}
$$

The first term inside first the square bracket in $K_{1}$ is the leading order fixed coupling kernel. The whole first square bracket in $K_{1}$ corresponds to the "Balitsky" running coupling prescription often 
used in LO phenomenology $[13,14]$, with the second square bracket being a pure NLO term. The coupling constant in front of the purely NLO kernels $K_{2}$ and $K_{f}$ is taken to depend on the parent dipole size $r$ and the explicit expressions for the kernels are

$$
\begin{aligned}
K_{2}= & -\frac{2}{\left(z-z^{\prime}\right)^{4}}+\left[\frac{X^{2} Y^{\prime 2}+X^{\prime 2} Y^{2}-4 r^{2}\left(z-z^{\prime}\right)^{2}}{\left(z-z^{\prime}\right)^{4}\left(X^{2} Y^{\prime 2}-X^{\prime 2} Y^{2}\right)}\right. \\
& \left.+\frac{r^{4}}{X^{2} Y^{\prime 2}\left(X^{2} Y^{\prime 2}-X^{\prime 2} Y^{2}\right)}+\frac{r^{2}}{X^{2} Y^{\prime 2}\left(z-z^{\prime}\right)^{2}}\right] \ln \frac{X^{2} Y^{\prime 2}}{X^{\prime 2} Y^{2}} \\
K_{f}= & \frac{2}{\left(z-z^{\prime}\right)^{4}}-\frac{X^{\prime 2} Y^{2}+Y^{\prime 2} X^{2}-r^{2}\left(z-z^{\prime}\right)^{2}}{\left(z-z^{\prime}\right)^{4}\left(X^{2} Y^{\prime 2}-X^{\prime 2} Y^{2}\right)} \ln \frac{X^{2} Y^{\prime 2}}{X^{\prime 2} Y^{2}} .
\end{aligned}
$$

In addition to the $\beta$-function terms, two kinds of logarithms appear in the kernels. The ones in $K_{2}$ and $K_{f}$ depend on conformal ratios of four distances, and vanish in the limit $r \rightarrow 0$. The first kernel $K_{1}$, on the other hand, has a nonconformal double logarithm that diverges in the limit $r \rightarrow 0$. Although this is an integrable singularity in the $z$-integral, it nevertheless has a problematic effect on the evolution equation, as we will show in the following. As an initial condition we use a parametrization

$$
N(r) \equiv 1-S(r)=1-\exp \left[-\frac{\left(r^{2} Q_{\mathrm{s} 0}^{2}\right)^{\gamma}}{4} \ln \left(\frac{1}{r \Lambda_{\mathrm{QCD}}}+e\right)\right]
$$

with two tunable parameters:

- The ratio $Q_{\mathrm{s} 0} / \Lambda_{\mathrm{QCD}}$ essentially determines value of $\alpha_{\mathrm{s}}$ and controls the overall relative importance of the NLO corrections.

- The anomalous dimension $\gamma$ controls the shape of the initial condition. Leading order fits to HERA data using the parametrization (2.5) prefer a value $\gamma \gtrsim 1$ which then becomes $\gamma \sim 0.8$ during the BK evolution. Since in an NLO fit also the impact factor relating the cross section and the dipole amplitude $N(r)$ should be different from the LO one, without performing the full fit it is not a priori obvious what would be a value favored by experimental data.

\section{Properties of the solution}

Figure 2 (left) shows the logarithmic evolution speed $\partial_{y} N(r) / N(r)$ for the MV model initial condition $\gamma=1$. Firstly it is obvious that for small values of $Q_{\mathrm{S}} / \Lambda_{\mathrm{QCD}}$, i.e. effectively large couplings, the the evolution speed is negative at all values if $r$. This means that the NLO corrections are large and negative, and the scattering amplitude actually decreases with energy; a very nonintuitive result. For smaller typical values of $\alpha_{\mathrm{s}}$ the region around the "front" $r \sim 1 / Q_{\mathrm{s}}$ behaves in a reasonable way, but for very small dipoles the evolution speed still diverges as $\partial_{y} N / N \sim \ln r$. Figure 2 (right) shows the contributions from different parts of the equation. The LO term gives a positive $\partial_{y} N / N$ that approaches a constant at $r \rightarrow 0$. The divergence for small $r$ is due to the nonconformal double logarithm, while the other NLO corrections yield a contribution that is negative, but smaller in magnitude than the leading order result.

While having $\partial_{y} N(r)<0$ or $N(r)<0$ is not very physical, it is not in itself a mathematical contradiction. A divergent $\partial_{y} N / N \sim \ln r$ in the limit $r \rightarrow 0$ is, however, a signal of an instability in 

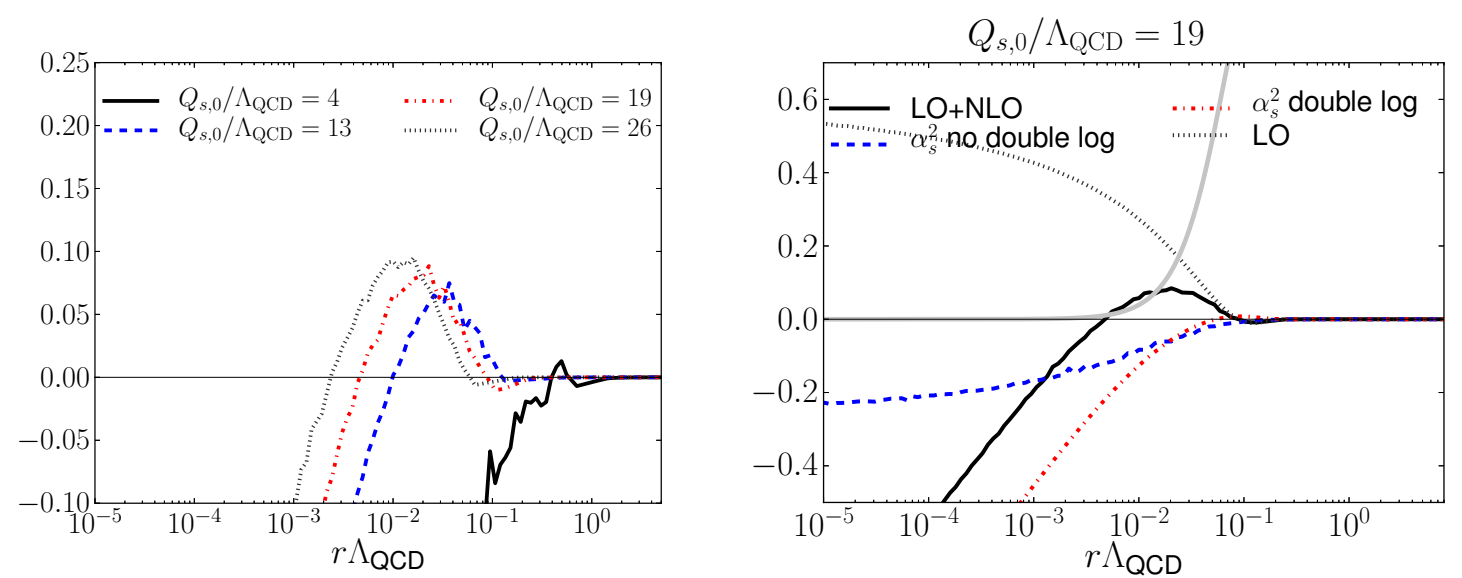

Figure 2: Left: Evolution speed $\partial_{y} N(r) / N(r)$ at the initial condition $y=0$ for the MV model initial condition, $\gamma=1$ Right: Contribution of different parts of the equation (2.1) to the evolution speed $\partial_{y} N(r) / N(r)$. Also the dipole amplitude $N(r)$ is shown with a thick grey line.

the system. One way to see this is the following simple argument. Let us consider a small but finite interval in rapidity, $\mathrm{d} y$, as in a numerical solution of the evolution equation. A diverging $\partial_{y} N / N$ for $r \rightarrow 0$ means that there is a small but finite $r$ below which $N$ becomes negative already in this one step in rapidity. This immediately makes the equation unstable, since the convergence of the $z$-integral of the leading order equation requires $N(r) \rightarrow 0$ for $r \rightarrow 0$. A finite $N(r=0)$ is also inconsistent with the definition $N(x-y)=1-\frac{1}{N_{\mathrm{c}}} \operatorname{Tr} U^{\dagger}(x) U(y)$ in terms of Wilson lines. To avert this problem in the numerics, we always enforce $N(r) \geq 0$ by hand.

\section{The anomalous dimension}
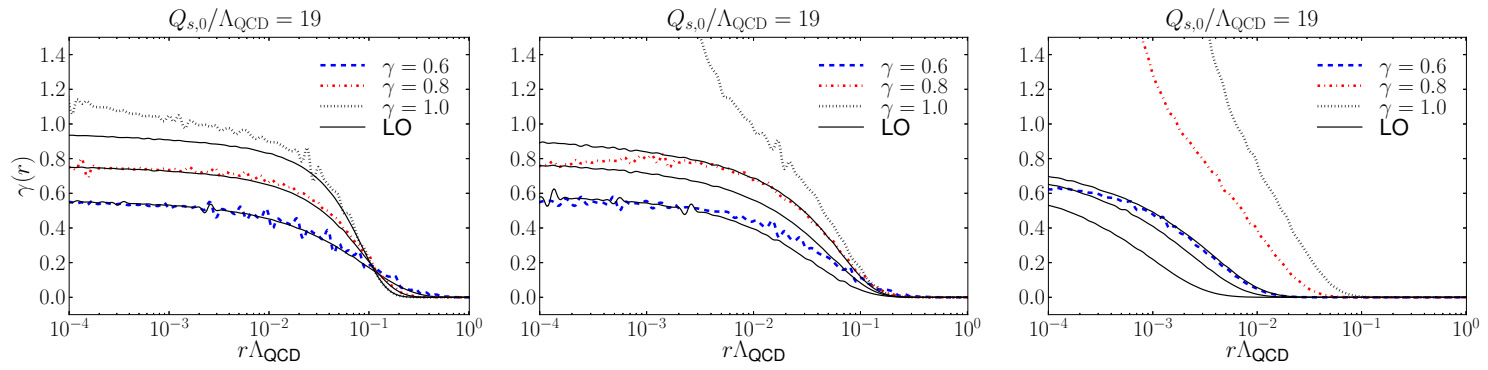

Figure 3: Anomalous dimension $\gamma(r)=\mathrm{d} \ln N(r) / \mathrm{d} \ln r^{2}$ at $y=1,5,30$.

Some more insight into the behavior of the equation can be obtained by following the development of the $r$-dependent anomalous dimension that we define here as

$$
\gamma(r)=\frac{\mathrm{d} \ln N(r)}{\mathrm{d} \ln r^{2}}
$$

In terms of the anomalous dimension we can discuss the behavior of the equation at small $r$ by parametrizing the amplitude as $N(r) \sim\left(Q_{\mathrm{s}} r\right)^{2 \gamma}$. For an appropriate $\gamma$ the leading order equation 
maintains this form, with $Q_{\mathrm{s}}^{2} \sim e^{\lambda y}$ and $\partial_{y} N / N \approx 2 \gamma \lambda>0$. If, due to large NLO corrections, one has $\partial_{y} N / N \rightarrow-c<0$ for $r \rightarrow 0$, this means that the solution behaves as $N \sim e^{-c y}$ : mathematically this is not problematic, but physically it is unnatural to have the scattering amplitude decrease with energy. If, however, $\partial_{y} N / N \sim c \ln r$, we can parametrize $N(r) \sim\left(Q_{s} r\right)^{2 \gamma(y)}$, with $\gamma(y) \sim y$. In other words, for a diverging evolution speed the functional form of the amplitude as a function of $r$ gets steeper with the evolution. If one enforces $N>0$ for small $r$ and $N<1$ for $r \rightarrow \infty$, the amplitude eventually develops into a discontinuous form $N(r) \sim \theta\left(r-1 / Q_{\mathrm{s}}\right)$. The evolution of $\gamma(r)$ for different initial conditions is shown in Fig. 3, where this behavior can be clearly seen. The unstable nature of the equation shows up as an increase in $\gamma(r)$ for small $r$. This increase starts immediately for the initial condition with $\gamma=1$, more slowly for the initial condition $\gamma=0.8$ and is not noticeable within the rapidity range studied here for $\gamma=0.6$.

\section{The composite conformal dipole}

In Ref. [15] Balitsky and Chirilli note that the nonconformal double logarithmic term appears also in the equation for the conformal $N=4$ Super Yang-Mills theory. It is therefore interpreted as an artefact of a cutoff that breaks conformal invariance. The authors then propose to correct for this effect order by order in perturbation theory by introducing a "composite conformal dipole" operator defined as

$$
S(r)^{\mathrm{conf}}=S(r)-\frac{\alpha_{\mathrm{s}} N_{\mathrm{c}}}{4 \pi^{2}} \int \mathrm{d}^{2} z \frac{r^{2}}{X^{2} Y^{2}} \ln \frac{a r^{2}}{X^{2} Y^{2}}[S(X) S(Y)-S(r)],
$$

with $a$ a dimensionful constant that drops out of the final equation. Rewriting the equation (2.1) in terms of the conformal dipole (5.1) removes the nonconformal double logaritm, but introduces an additional term in the other kernel $K_{2}$ that behaves as $\ln r^{2}$ for small $r$. We have checked numerically that the qualitative behavior of the conformal dipole equation remains the same as the original one. As shown in Fig. 4, it is now this new $\ln r^{2}$ term which is responsible for the leading behavior at small $r$.

In conclusion, we have performed the first numerical solution of the full NLO BK equation directly in coordinate space. The

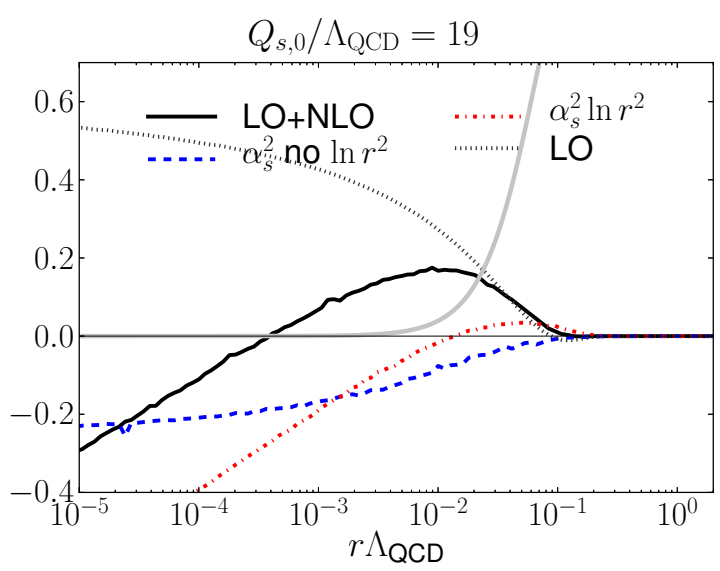

Figure 4: Contribution of different terms to the evolution speed $\partial_{y} N / N$ of the conformal dipole. NLO corrections are negative, implying a slower energy dependence of cross sections than with the LO equation. This generically leads to a better agreement with experimental data. The equation has, however, a double logaritmic term that causes a problematic behavior for small dipoles, i.e. high $Q^{2}$. It seems evident that these large logarithms will need to be resummed in order for the equation to be useful in practical phenomenological work. 
Acknowledgements This work has been supported by the Academy of Finland, projects 267321 and 273464, the Graduate School of Particle and Nuclear Physics (H.M.) and by computing resources from CSC - IT Center for Science in Espoo, Finland.

\section{References}

[1] F. Gelis, E. Iancu, J. Jalilian-Marian and R. Venugopalan, The color glass condensate, Ann. Rev. Nucl. Part. Sci. 60 (2010) 463 [arXiv: 1002.0333 [hep-ph] ].

[2] I. Balitsky, Operator expansion for high-energy scattering, Nucl. Phys. B463 (1996) 99 [arXiv:hep-ph/9509348].

[3] Y. V. Kovchegov, Small-x F2 structure function of a nucleus including multiple pomeron exchanges, Phys. Rev. D60 (1999) 034008 [arXiv: hep-ph/9901281].

[4] I. Balitsky and G. A. Chirilli, Next-to-leading order evolution of color dipoles, Phys. Rev. D77 (2008) 014019 [arXiv:0710.4330 [hep-ph]].

[5] I. Balitsky and G. A. Chirilli, Rapidity evolution of Wilson lines at the next-to-leading order, Phys. Rev. D88 (2013) 111501 [arXiv:1309.7644 [hep-ph] ].

[6] A. Kovner, M. Lublinsky and Y. Mulian, Jalilian-Marian, Iancu, McLerran, Weigert, Leonidov, Kovner evolution at next to leading order, Phys. Rev. D89 (2014) 061704 [arXiv: 1310.0378 [hep-ph]].

[7] I. Balitsky and G. A. Chirilli, Photon impact factor in the next-to-leading order, Phys. Rev. D83 (2011) 031502 [arXiv:1009.4729 [hep-ph]].

[8] G. Beuf, NLO corrections for the dipole factorization of dis structure functions at low x, Phys. Rev. D85 (2012) 034039 [arXiv:1112.4501 [hep-ph]].

[9] G. A. Chirilli, B.-W. Xiao and F. Yuan, One-loop factorization for inclusive hadron production in $p A$ collisions in the saturation formalism, Phys. Rev. Lett. 108 (2012) 122301 [arXiv:1112. 1061 [hep-ph] ].

[10] T. Altinoluk, N. Armesto, G. Beuf, A. Kovner and M. Lublinsky, Single-inclusive particle production in proton-nucleus collisions at next-to-leading order in the hybrid formalism, Phys. Rev. D91 (2015) 094016 [arXiv:1411.2869 [hep-ph]].

[11] T. Lappi and H. Mäntysaari, Direct numerical solution of the coordinate space Balitsky-Kovchegov equation at next to leading order, Phys. Rev. D91 (2015) 074016 [arXiv: 1502.02400 [hep-ph]].

[12] I. Balitsky, Quark contribution to the small-x evolution of color dipole, Phys. Rev. D75 (2007) 014001 [arXiv:hep-ph/0609105].

[13] J. L. Albacete and C. Marquet, Single inclusive hadron production at RHIC and the LHC from the color glass condensate, Phys. Lett. B687 (2010) 174 [arXiv:1001.1378 [hep-ph] ].

[14] T. Lappi and H. Mäntysaari, Single inclusive particle production at high energy from HERA data to proton-nucleus collisions, Phys. Rev. D88 (2013) 114020 [arXiv:1309.6963 [hep-ph]].

[15] I. Balitsky and G. A. Chirilli, NLO evolution of color dipoles in N=4 SYM, Nucl. Phys. B822 (2009) $45[\operatorname{arXiv}: 0903.5326$ [hep-ph]]. 